\title{
Behçet's Disease with Multiorgan Infection: Alternative Treatment to Systemic Immunosuppressants
}

\section{Dian Hasanah ${ }^{1}$}

${ }^{1}$ Department of Internal Medicine, Faculty of Medicine, Universitas Brawijaya, Malang, West Java, Indone sia

\author{
A R T I C L E I N F O \\ Keywords: \\ Behçet disease \\ Emphysematous Lungs \\ Infection \\ Colchicine \\ Rebamipide \\ Sucralfate

\section{Corresponding author: \\ Dian Hasanah} \\ E-mail addre ss: \\ dianhasanah.fk@ub.ac.id
}

All author have reviewed and approved the final version of the manuscript.

https://doi.org/10.37275/IJR.v12i2.122

\begin{abstract}
A B S T R A C T
Background. Behçet's disease is a rare systemic autoimmune vasculitis. The presence of infection makes it difficult to manage. Case. Ms. X, 19 years old, complained of mouth and genitalia ulcers and joints pain. She had typhoid fever two months before; and a week after recove ry, she experiencedreddish skin lesions on her legs. Her condition was weak and the pain visual analog score (VAS) was 9/10. Multiple oral ulcers were found in her oral cavity and vulva. Her lungs were hypersonor. There were hyperpigmented maculae in her lower extremities. Her ankle joints were painful in passive and active movements. Blood leukocytes: 13,210/ $\mu$ l, urine leukocytes: 20-23/hpf, ANA, anti-dsDNA and Pathergy tests were negative. The diagnosis of Behçet's disease was based on multiple oral and genital ulcers, history of erythema nodosum and arthritis. The patient was treated with topical triamcinolone. Joint ulcers and pain worsened; so that intravenous methylprednisolone was administer. Two days later, the patient had cough and oxygen desaturation. Chest X-ray showed emphysematous lungs and pneumonia. Methylprednisolone was stopped, ceftriaxone and levofloxacin were given, treatment for ulcers and arthritis was replaced with colchicine and rebamipide. Sucralfate is given for gargle and applied to the genital ulcers. On the seventh day, the ulcers were greatly reduced, joint pain resolved, cough subsided and VAS became 2/10. On the twelfth day, the chest X-ray was normal and ulcers were healed, ankle pain was suffered again accompanied by swelling. The patient was treated with methylprednisolone and azathioprine. Discussion. Treatment for this disease is immunosuppressants. In severe conditions with infection, colchicine can be an alternative treatment. Adjuvant treatment are rebamipide and/or sucralfate. Emphysematous lungs can be caused by alpha-1 antitrypsin deficiency or pulmonary vasculitis, which can be manifested in autoimmune diseases. Emphysematous lungs, leads us to follow up on the development to other autoimmune disorders such as lupus. Conclusion. Behçet's disease with infection can be successfully managed with colchicine, rebamipide and sucralfate.
\end{abstract}

\section{Introduction}

Behcet's disease is a rare disorder that causes blood vessel inflammation throughout body. The disease can lead to numerous signs and symptoms, include mouth sores, eye inflammation, skin rashes and lesions, and genital sores. Treatment involves medications to reduce the signs and symptoms of Behcet's disease and to prevent serious complications, such as blindness. Steroids and immunosuppressive agents are drugs of choice. The presence of infections makes the management become dilemmatic.

\section{Case}

Ms. D, 19 years old, a student and unmarried, complained of painful mouth ulcers for two weeks which grew larger in number and size until she had difficulty in eating. She also experienced painful genital ulcers and joints pain in both knee and ankle without swelling. Two months before, she was hospitalized in a private hospital because of fever, diarrhea and vomiting, and was diagnosed with typhoid fever. A week after recovered from typhoid, she experienced painful reddish skin lesions, bulging 
in palpation, with a mean diameter around $1 \mathrm{~cm}$ in both upper and lower limbs, totally about 15 pieces. Skin lesions resolved with analgesic from doctors in Primary Health and left hyperpigmentation.

At admission, she looked weak, emaciated and antalgic gait. Blood pressure, pulse rate, respiratory rate and body temperature were normal. The pain visual analog score (VAS) was 9/10. She had shrunken eyes and decreased skin turgor. There were multiple and confluent oral ulcers, the largest size was $7 \times 7 \mathrm{~mm}$, involving the mucosa of oral cavity including the hard palate. Thorax percussion was hypersonor on both hemithorax. There were multiple vulvar ulcers with the largest size was $6 \times 6 \mathrm{~mm}$. On the extensor side of lower extremities, multiple hyperpigmented maculae were found at the location that was formerly the patient called a painful reddish lesions that can be suspected as erythema nodosum. Her ankles were warm and painful on passive and active movements, but no other signs of inflammation were found. There were no eyes complaints such as redness, pain, dazzled or blurred vision.

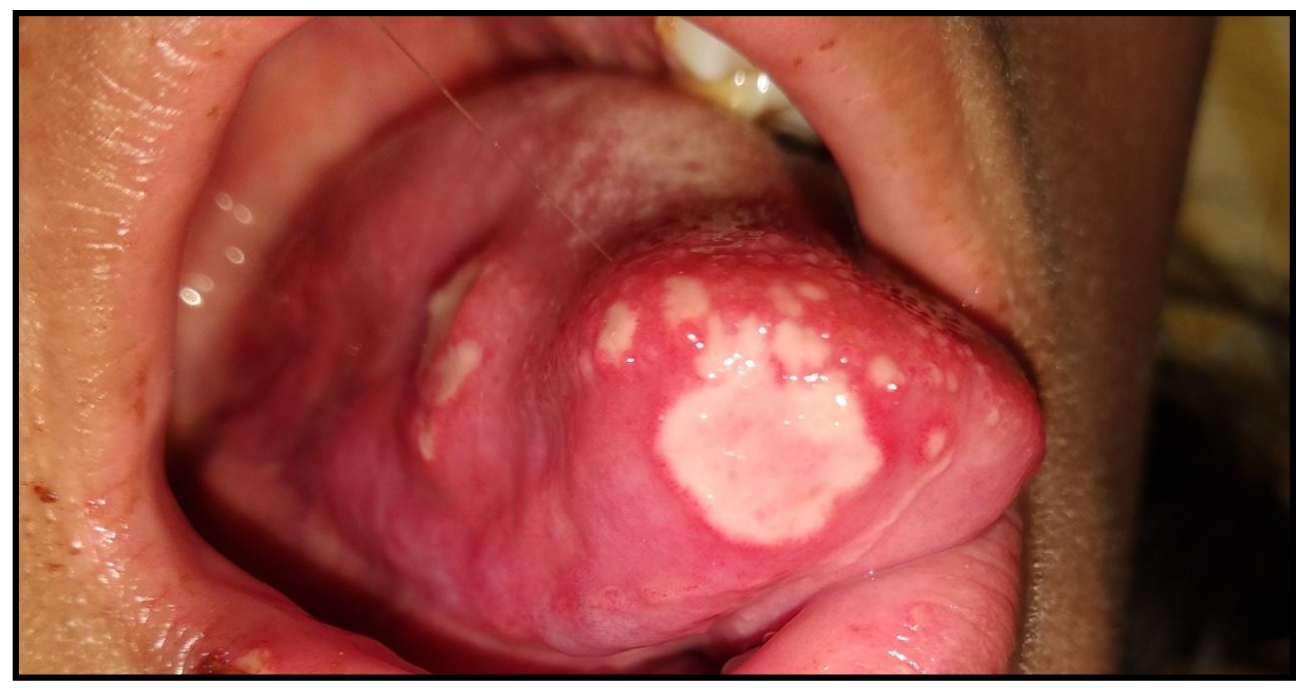

Figure 1. Oral ulcer before treatment.

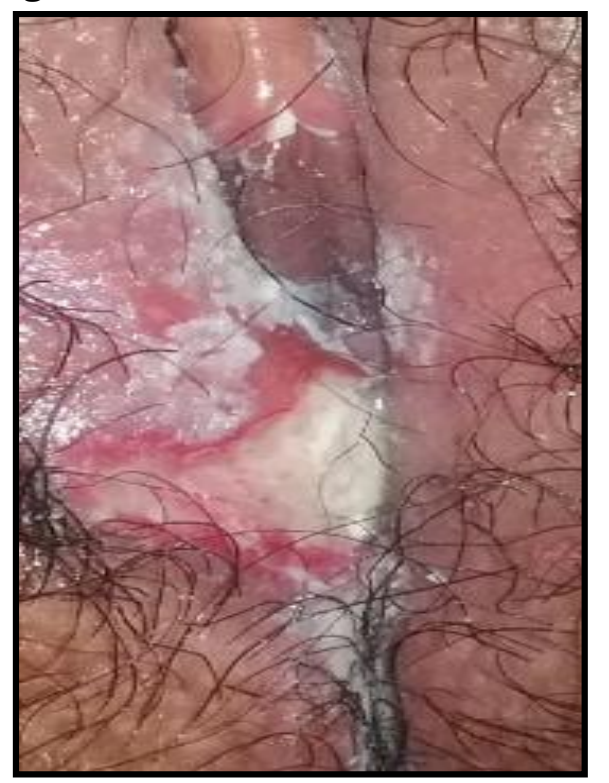

Figure 2. Genital ulcer before treatment. 

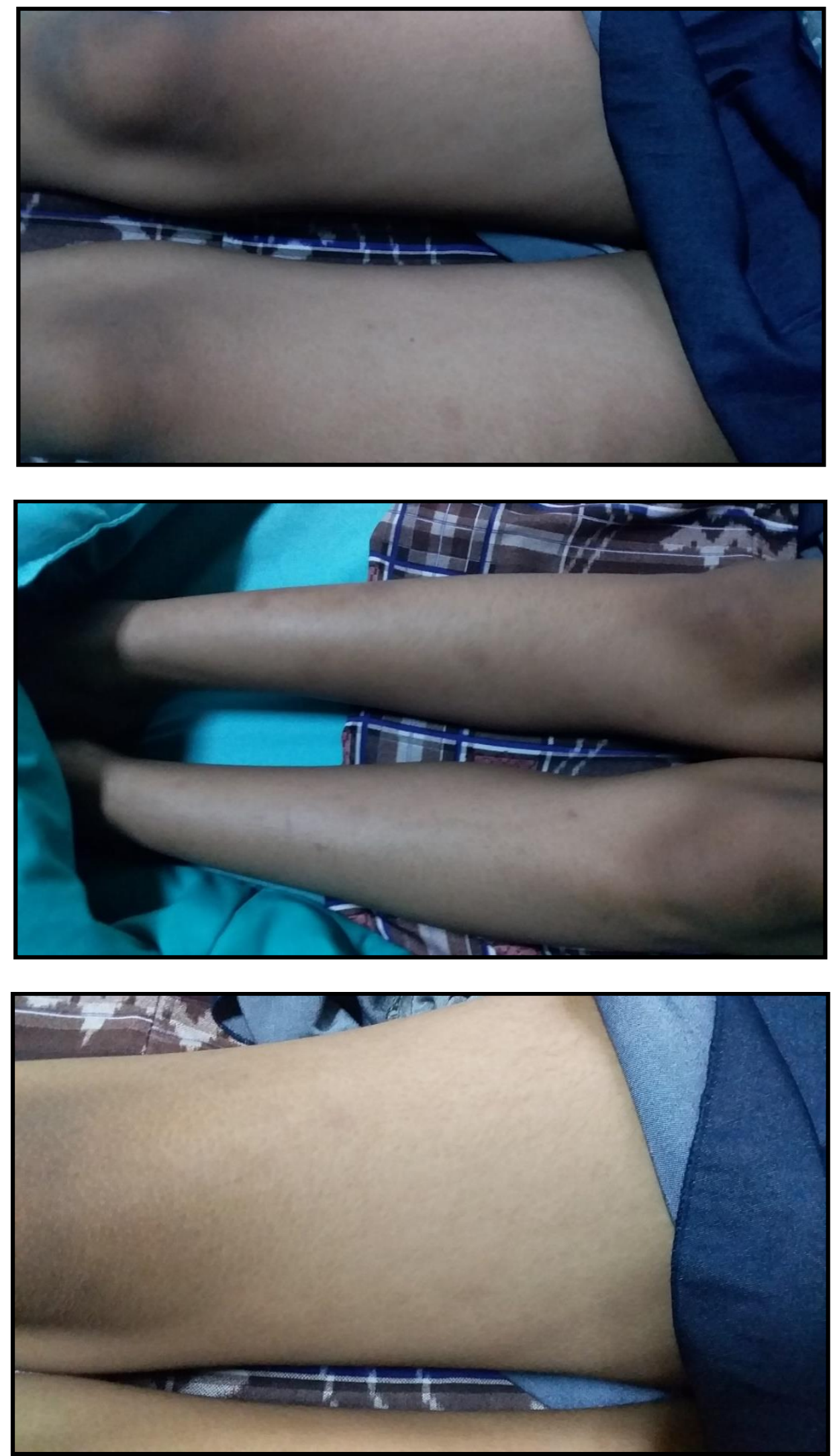

Figure 3. Hyperpigmentation on the extensor side of the lower extremities. 


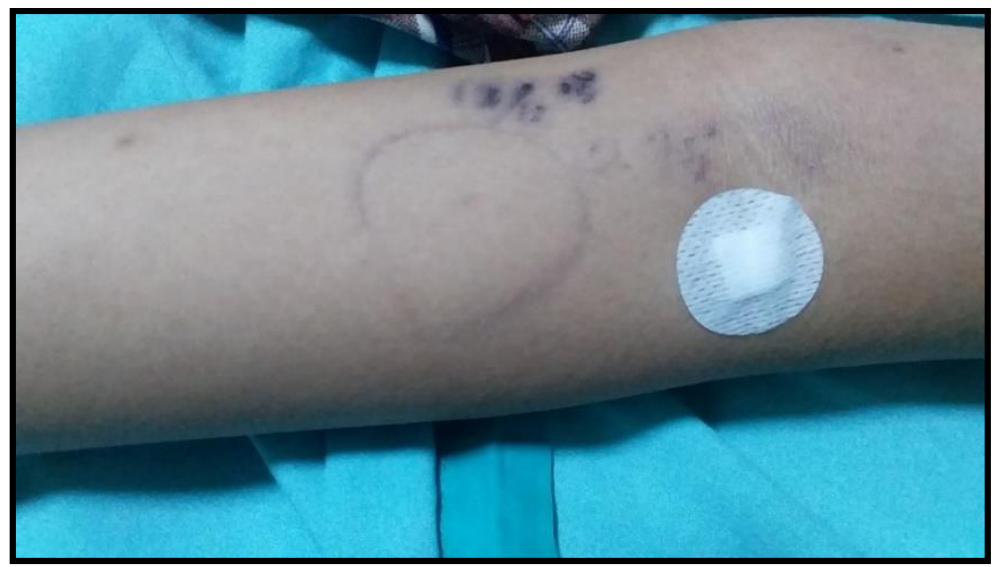

Figure 4. The Pathergy test showed negative result after 48 hours of observation.

Laboratory results were: blood leukocytes: 13,210/ $\mu 1$, platelets: $648,000 / \mu 1$, urine leukocytes: 20-23/hpf, ANA test: 0.4 ratio (negative), anti-dsDNA IgM: $9.9 \mathrm{IU} / \mathrm{ml}$ (negative) and IgG anti-dsDNA: 5.1 $\mathrm{IU} / \mathrm{ml}$ (negative). Pathergy test was negative. The diagnosis of Behçet's disease was based on: multiple oral and genital ulcers, history of erythema nodosum and arthritis.

The patient was given topical triamcinolone and topical analgesic for ulcers, intravenous ceftriaxone and methampyrone. Within a day of evaluation, the ulcers and joint pain worsened, so intravenous methylprednisolone was administered. Patient was also treated with oral nystatin to prevent growth of fungi.
Two days later, patients complained cough with white sputum, oxygen saturation dropped to $92 \%$ and thorax X ray showed emphysematous lungs and pneumonia. Methylprednisolone were discontinued. The combination of ceftriaxone and levofloxacin was given to treat pneumonia and asymptomatic urinary tract infections. Ulcer and arthritis treatment was replaced with colchicine tablets 2 x $0.6 \mathrm{mg}$. Topical triamcinolone and methampyrone were continued. Sucralfate was given for gargle and applied to the genital ulcers. On the seventh day, oral and genital ulcers are greatly reduced, joints pain resolved and VAS becomes 2/10. Blood tests showed normal leukocytes, but lymphopenia $(1,063 / \mu 1)$ was revealed. The patient continued treatment in outpatient care.

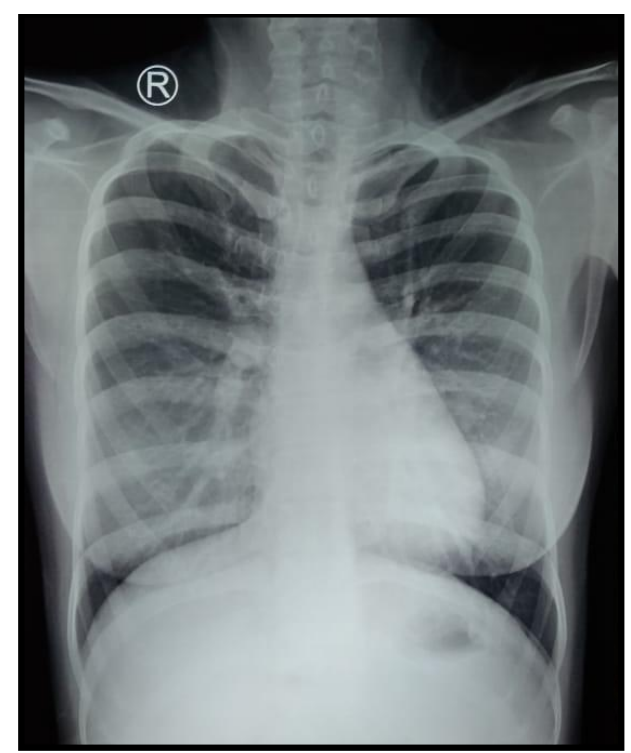

Figure 5. Thorax X-ray before treatment showed emphysematous lungs and right middle and lower infiltrates. 


\section{Outcome}

Thorax X-ray at on the 12th day was normal, the patient did not complain cough. Antibiotics were continued for three days later. All oral mucosa has been completely resolved and the size of the genital ulcer has diminished. Joints pain was complained again, accompanied with swelling in both ankles. Patients were still treated with colchicine.
Immunosuppressants were postponed with consideration of restoring urinary tract infections and pneumonia. At the evaluation of the 20th day, joints inflammation was still occurred. Methylprednisolone $3 \times 8 \mathrm{mg}$ and azathioprine $2 \times 50 \mathrm{mg}$ were started. Until now, she is under controlled by these medications.

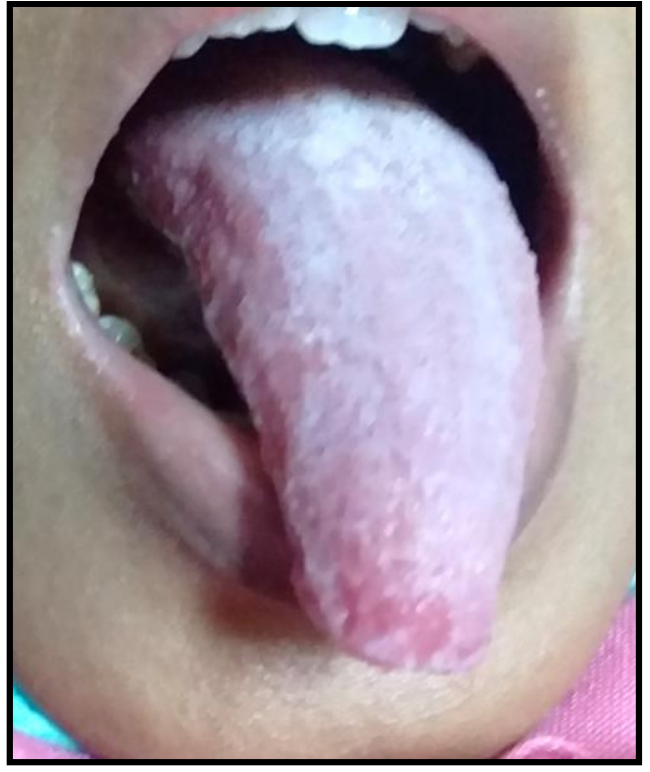

Figure 6. Oral ulcer on the 12 th day after treatment, the oral mucosa resolved to normal.

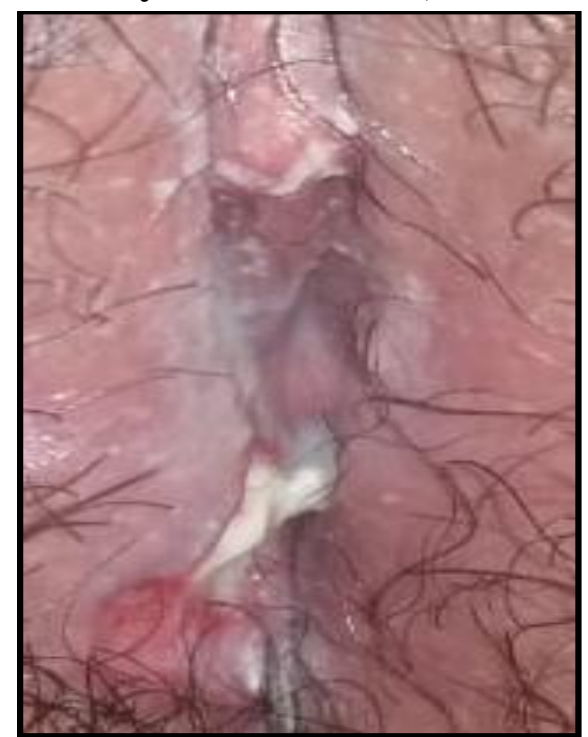

Figure 7. Genital ulcer on the 12 th day after treatment, the size diminished. 


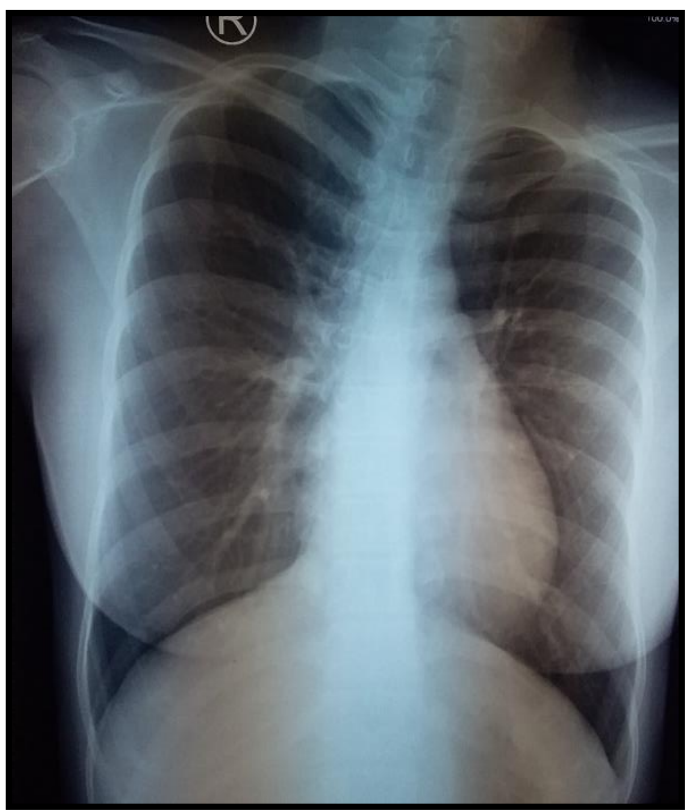

Figure 8. Thorax X-ray on the 12th day after treatment, infiltrates diminished.

\section{Discussion}

Behçet's disease is a rare autoimmune disease with inflammatory of blood vessels (vasculitis) as manifestation, characterized by oral and genital ulcers, uveitis and skin lesions that can undergo remission and exacerbations. 1 This disease often involves joints, gastrointestinal tract, lungs and nervous system. One of the potential etiologies of this disease is a previous bacterial infection, one of them Salmonella spp. ${ }^{2}$

Treatment for this disease is using steroids or other immunosuppressant drugs, can be used topically or systemically with consideration of disease severity.1,2 If the condition is severe but there are other conditions which are dilemmatic for systemic immunosuppressant administration, such as the presence of infection, colchicine can be an alternative. 2,3 Colchicine is an anti-inflammatory, which its actions are: inhibits the surface expression of adhesion molecules in T cells and endothelial cells, inhibits monocyte migration, decreases the secretion of matrix metalloproteinase- 9 and inhibits the synthesis of tumor necrosis factor and interleukin-6. Colchicine also binds to platelet microtubules which will inhibit platelet aggregation and secretion. ${ }^{3}$

Adjuvant treatment that can be considered are rebamipide and sucralfate, which has been proven from various studies could correct oral and genital ulcers.4,5 Rebamipide helps replace tissue loss by increasing the expression of epidermal growth factor (EGF) and EGF receptors. This EGF will cause angiogenesis and improve tissue granulation and epithelialization for ulcer healing. 4 Meanwhile, sucralfate will bind to the ulcer and form a barrier to accelerate ulcer healing. ${ }^{5}$ This disease can progressively affect many organs such as the eyes (uveitis) and the nervous system and can also be recurrent. ${ }^{1}$

The emphysematous lungs of the patient, which is an unusual condition for young women without exposure to cigarettes and noxious gas, might be related to autoimmune disease. Emphysematous lungs can occur due to alpha-1 antitrypsin deficiency, which can be found in patients with autoimmune diseases, one of which is associated with Behçet's disease.6,7 The pathogenesis is pulmonary vasculitis, and it is well known that the pathomechanism of Behçet's disease is vasculitis. ${ }^{8}$ Emphysematous lungs can also be a clue to poor prognosis of the patient. In addition, follow-up is also needed to prevent systemic vascular thrombosis due to vasculitis. 


\section{Conclusion}

Steroids and immunosuppressive agents are the drugs of choice for Behçet's disease. The presence of infections makes the management become dilemmatic, because they can worsen the infections. Colchicine, rebamipide and sucralfate can be given to control the disease while waiting for the infection to subside.

\section{References}

1. Salmaninejad A, Gowhari A, Hosseini S, Aslani $\mathrm{S}$, Yousefi M, Bahrami T et al. Genetics and Immunodysfunction Underlying Behçet's Disease and Immunomodulant Treatment Approaches. J Immunotoxico. 2017;14(1):137 151.

2. Hatemi G, Christensen R, Bang D, Bodaghi B, Chamberlain AM, Gul A et al. 2018 Update of the EULAR Recommendations for the
Management of Behçet's Syndrome. Ann Rheum Dis. 2018;77:808-818.

3. Atas H, Cemil BC, Canpolat F, Gönül M. The Effect of Colchicine on Mean Platelet Volume in Behcet's Disease. Ann Clin Lab Sci. 2015;45(5):545-549.

4. Kudur MH, Hulmani M. Rebamipide: A Novel Agent in the Treatment of Recurrent Aphthous Ulcer and Behcet's Syndrome. Indian J Dermatol. 2013;58(5):352-354.

5. Rees W. Mechanisms of Gastroduodenal Protection by Sucralfate. Am J Med. 1991;91(2A):58S-63S

6. de Serres F, Blanco I. Role of Alpha-1 Antitrypsin in Human Health and Disease (Review). J Intern Med. 2014;276:311-335.

7. Song S. Alpha-1 Antitrypsin Therapy for Autoimmune Disorders. Chronic Obstr Pulm Dis. 2018;5(4):289-301.

8. Bilgin G, Sungur G, Kucukterzi V. Systemic and Pulmonary Screening of Patients with Behçet's Disease During Periodic Follow-up. Respir Med. 2013;107(3):466-4 